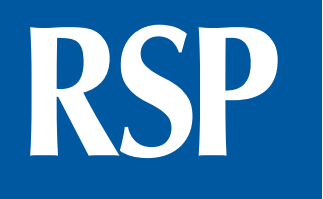

http://www.rsp.fsp.usp.br/
Revista de Saúde Pública

\title{
Inatividade física no lazer e na escola está associada à presença de transtornos mentais comuns na adolescência
}

\author{
Vanessa Roriz Ferreira' (iD, Thiago Veiga Jardim ${ }^{1,1,1,111}$ (iD), Thaís Inácio Rolim Póvoa ${ }^{\mathrm{IV}}$ (iD, Ricardo \\ Borges Viana'v (iD, Ana Luiza Lima Sousa' iD, Paulo César Veiga Jardim' iD \\ ' Universidade Federal de Goiás. Faculdade de Medicina. Liga de Hipertensão Arterial. Goiânia, GO, Brasil \\ " Brigham \& Women's Hospital. Division of Cardiovascular Medicine. Boston, MA, USA \\ III Harvard TH Chan School of Public Health. Department of Health Policy and Management. Center for Health \\ Decision Science, Boston, MA, USA \\ iv Universidade Estadual de Goiás. Laboratório de Fisiologia do Exercício da Faculdade do Esporte ESEFFEGO. \\ Goiânia, GO, Brasil
}

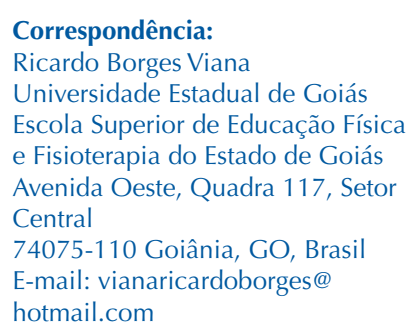

Como citar: Ferreira VR, Jardim TV, Póvoa TIR, Viana RB, Sousa ALL, Jardim PCV. Inatividade física no lazer e na escola está associada à presença de transtornos mentais comuns na adolescência. Rev Saude Publica. 2020;54:128.

Copyright: Este é um artigo de acesso aberto distribuído sob os termos da Licença de Atribuição Creative Commons, que permite uso irrestrito, distribuição e reprodução em qualquer meio desde que o autor e a fonte originais sejam creditados.

\section{RESUMO}

OBJETIVO: Investigar a associação da inatividade física no lazer e na escola com os transtornos mentais comuns durante a adolescência.

MÉTODOS: A amostra foi composta por 73.399 adolescentes (12-17 anos), participantes do Estudo de Riscos Cardiovasculares em Adolescentes (Erica). Esse estudo transversal, nacional e de base escolar foi realizado em 2013 e 2014, nos municípios brasileiros com mais de 100 mil habitantes. A atividade física no lazer foi categorizada de acordo com o volume de prática semanal, e os adolescentes foram classificados em ativos ( $\geq 300$ minutos/semana), inativos (0 minuto/semana) e insuficientemente ativos (1-299 minutos/semana). A prática esportiva e a participação em aulas de educação física na escola também foram analisadas. A presença de transtornos mentais comuns foi avaliada a partir do general health questionnaire, com ponto de corte maior ou igual a 3. Odds ratios (OR) foram estimados utilizando regressão logística múltipla.

RESULTADOS: A chance de transtornos mentais comuns foi 16\% maior no grupo que relatou ser inativo (0 minuto/semana) no lazer (OR = 1,16; IC95\% 1,06-1,27). A prevalência de transtornos mentais comuns foi maior entre jovens que não praticaram esportes (37,2\% vs. 25,9\%; OR = 1,14; IC95\% 1,04-1,25) e não participaram das aulas de educação física na escola (39,5\% vs. 29,6\%; OR = 1,25; IC95\% 1,15-1,36). Atividade física insuficiente (1-299 minutos/semana) não aumentou a razão de chances de transtornos mentais comuns. Praticar atividade física no lazer, independentemente da duração e frequência semanal, reduziu em $26 \%$ as chances de transtornos mentais comuns nessa população.

CONCLUSÕES: A inatividade física no lazer e na escola foi associada à presença de transtornos mentais comuns na adolescência. Os resultados sugerem que prática esportiva, educação física escolar e atividade física durante o lazer, mesmo sem atingir a recomendação atual, têm relação com a saúde mental dos jovens.

DESCRITORES: Adolescente. Comportamento Sedentário. Transtornos Mentais. Saúde Mental. Educação Física e Treinamento. 


\section{INTRODUÇÃO}

Dados epidemiológicos mostram que até $20 \%$ das crianças e adolescentes sofrem de uma doença mental incapacitante e que cerca de $50 \%$ dos transtornos mentais têm início na adolescência ${ }^{1}$. Mundialmente, os transtornos neuropsiquiátricos são a principal causa de anos de vida perdidos por incapacidade na faixa etária entre 10 e 24 anos $^{2}$. No Brasil, dados do Estudo de Riscos Cardiovasculares em Adolescentes (Erica) relataram elevada prevalência $(30,0 \%)$ de transtornos mentais comuns na população jovem³ .

Os transtornos mentais comuns, também conhecidos como distúrbios psiquiátricos menores ou sofrimento psíquico, são desordens mentais sem caráter psicótico não contempladas nos critérios diagnósticos dos sistemas de classificação categórica. São caracterizados principalmente pela presença de sintomas de depressão e ansiedade, além de queixas somáticas inespecíficas. Esses transtornos, que podem ser manifestações iniciais de doenças psiquiátricas mais graves, prejudicam a vida escolar e as relações sociais do adolescente $e^{4,5}$.

Evidências recentes relacionaram sintomas de depressão e ansiedade com inatividade física ${ }^{6}$. Estudo transversal com adolescentes australianos mostrou que meninos com baixo nível de atividade física estão mais propensos à sintomatologia depressiva ${ }^{6}$. Por outro lado, pesquisa longitudinal conduzida na Alemanha revelou que mulheres jovens (12-26 anos), ao praticarem atividade física por mais tempo que o usual, sentiam-se menos deprimidas na manhã do dia seguinte?

Ser ativo durante a adolescência também aumenta a chance de se manter ativo na idade adulta, previne doenças cardiovasculares, melhora a composição corporal e aumenta a força muscular, além dos benefícios imediatos ao jovem, tais como sensação de bem-estar e maior autoestima ${ }^{8}$. Apesar disso, no Brasil, um em cada quatro adolescentes não pratica nenhuma atividade física no lazer (0 min/semana) ${ }^{9}$. Este fato é preocupante, pois o estilo de vida sedentário na vida adulta é a quarta maior causa de mortes no mundo ${ }^{10}$.

Adicionalmente, a vida na adolescência é marcada por intensas transformações, tanto fisiológicas quanto sociais. É possível que ocorram trabalhos escolares de maior complexidade, separação dos pais e maior exigência da sociedade referente a planos futuros. Essa complexidade de fatores pode afetar o desejo ou a capacidade do adolescente de ser fisicamente ativo na vida diária, bem como intensificar seu transtorno mental comum ${ }^{1}$.

Até a data, a maioria das pesquisas tem focado em populações adultas de países desenvolvidos, nos comportamentos sedentários (tempo em atividades de tela) e nos portadores de enfermidades psiquiátricas. Além disso, pouco se sabe sobre a educação física escolar e a prática esportiva no contexto dos transtornos mentais comuns ${ }^{11}$. Nesse sentido, o presente estudo teve como objetivo investigar a associação de inatividade física no lazer e na escola com transtornos mentais comuns em adolescentes escolares brasileiros.

\section{MÉTODOS}

O Estudo de Riscos Cardiovasculares em Adolescentes (Erica) é um estudo transversal, multicêntrico, nacional, de base escolar, que avaliou adolescentes com idades entre $12 \mathrm{e}$ 17 anos, matriculados em escolas públicas e privadas (período matutino e vespertino), de municípios brasileiros com mais de 100.000 habitantes. Os dados foram coletados entre março de 2013 e dezembro de 2014. O protocolo do Erica foi previamente descrito ${ }^{12}$.

A população da pesquisa foi dividida em 32 estratos geográficos, constituídos por 27 capitais e cinco conjuntos de municípios com mais de 100 mil habitantes em cada uma das cinco macrorregiões geográficas do país. Em seguida, realizou-se uma amostragem probabilística de escolas em duas etapas. Na primeira, foram selecionadas escolas para cada estrato geográfico, com probabilidade proporcional ao seu tamanho e inversamente 
proporcional à distância da capital. Na segunda etapa, três turmas em cada escola sorteada foram selecionadas com equiprobabilidade durante o trabalho de campo, usando o ano da turma como variável informativa da idade. A amostra é representativa para municípios de médio e grande porte (> 100 mil habitantes) em âmbito nacional e regional e para as capitais brasileiras.

Nas turmas selecionadas, foram entrevistados e examinados todos os alunos que assinaram o termo de assentimento e trouxeram o termo de consentimento livre e esclarecido assinado pelos responsáveis (quando exigido pelo Comitê de Ética em Pesquisa local). Foram excluídos das análises, por não serem considerados elegíveis, adolescentes fora da faixa etária eletiva, com deficiência física ou mental e grávidas.

O instrumento de coleta dos dados foi um questionário autopreenchível, aplicado em sala de aula, sob supervisão da equipe do estudo, por meio de um coletor eletrônico de dados individual e portátil PDA (personal digital assistant, modelo LG GM750Q). Para caracterização da amostra estudada, os adolescentes responderam a questões sobre sexo (masculino ou feminino), idade em anos completos (posteriormente categorizada: 12-14 e 15-17 anos), cor da pele declarada, tipo de escola (pública ou privada), maturação sexual (autoavaliação baseada em cinco estágios) ${ }^{13}$ e estado de peso, a partir das curvas de índice de massa corporal para idade propostas pela Organização Mundial de Saúde em 2007.

Para determinação do nível de atividade física dos adolescentes, foi utilizada uma adaptação do self-administered physical activity checklist, que consiste em uma lista de 24 modalidades e permite que o adolescente informe a frequência (dias) e o tempo (horas e minutos) que praticou, na última semana, alguma das atividades listadas ${ }^{14}$. A versão desse questionário utilizada no Erica foi validada em adolescentes brasileiros ${ }^{15}$. No cálculo do tempo total de atividade física, consideraram-se modalidades de intensidade moderada a vigorosa (maior ou igual a três equivalentes metabólicos [METs]).

O produto entre o tempo e a frequência em cada atividade e o somatório dos tempos obtidos foram calculados para determinar o nível de atividade física. Os adolescentes que acumularam pelo menos 300 minutos/semana de atividade física foram considerados ativos no lazer, com base no nível recomendado pela $\mathrm{OMS}^{16}$. Os indivíduos que não referiram qualquer prática de atividade física no lazer na semana anterior à pesquisa (0 minuto/semana) foram considerados inativos. E aqueles cujo tempo acumulado nos últimos sete dias foi maior que 0 e menor que 300 minutos foram denominados insuficientemente ativos ${ }^{17}$.

A participação nas aulas de educação física da escola (sim/não e número de tempos por semana) e a prática esportiva fora/dentro da escola (sim/não) também foram avaliadas. Das 24 modalidades avaliadas para determinação do nível de atividade física dos adolescentes, 12 foram consideradas modalidades esportivas: atletismo, natação, lutas (como judô e caratê), tênis, ginástica olímpica, futebol, futsal, futebol de praia, handebol, basquete, vôlei de quadra e vôlei de praia.

Os transtornos mentais comuns foram avaliados por meio do general health questionnaire (GHQ-12), um instrumento de rastreamento psiquiátrico 5 . O GHQ-12 avalia o estado de saúde mental e o sofrimento psíquico sem caráter psicótico, a partir de 12 questões referentes às duas semanas precedentes, com quatro opções de resposta (mais que de costume, o mesmo de sempre, menos que de costume, muito menos que de costume ou muito mais que de costume). Os escores dos itens individuais foram codificados por meio de uma escala dicotômica. As respostas com 1 ou 2 pontos indicaram ausência (0), e as respostas com 3 ou 4 pontos, presença (1). Em seguida, todos os itens foram somados. Usando esse método, o participante pôde alcançar de 0 a 12 pontos; escores de 3 ou mais ${ }^{3}$ indicaram a presença de transtornos mentais comuns, que refere-se a um estado de risco para o desenvolvimento de distúrbios psiquiátricos ${ }^{4}$. O GHQ-12 foi validado para a população brasileira, tendo uma entrevista psiquiátrica estruturada como padrão ouro ${ }^{18}$. 
Foram calculadas medidas de frequência relativa (prevalências) de escore igual ou maior que 3 para as diferentes categorias de tempo de atividade física no lazer (adolescentes ativos, insuficientemente ativos e inativos), para a prática esportiva (sim/não) e para a prática de educação física na escola (sim/não e por número de tempos/semana). Considerando o desenho amostral, odds ratios (OR), com seus respectivos intervalos de $95 \%$ de confiança (IC95\%), foram estimados por meio de regressão logística simples e ajustada por sexo, idade, tipo de escola, estágio de maturação sexual, índice de massa corporal, tabagismo (fumou pelo menos uma vez nos últimos trinta dias) e consumo de álcool (consumiu pelo menos um copo ou dose de bebida alcoólica nos últimos trinta dias). Utilizou-se o módulo survey do programa Stata versão 12.0 (StataCorp) para análise de dados de amostra complexa, uma vez que emprega estratificação e conglomeração em seus estágios de seleção.

O Erica foi realizado de acordo com os princípios da Declaração de Helsinque. O estudo foi aprovado pelos Comitês de Ética em Pesquisa da Universidade Federal do Rio de Janeiro (Processo 45/2008) e de cada unidade da federação.

\section{RESULTADOS}

Foram avaliados 73.399 adolescentes (71,7\% dos alunos elegíveis), de 1.247 escolas, em 124 municípios brasileiros. A cobertura nacional dos adolescentes elegíveis foi de $74,8 \%$ $(\mathrm{n}=40.675)$ no sexo feminino e $68,3 \%(\mathrm{n}=32.724)$ no sexo masculino. Não foram considerados elegíveis 364 escolares com deficiência física que inviabilizava as medidas e 215 grávidas, representando 0,6\% do total da amostra de 12 a 17 anos. A recusa de participação no estudo foi maior nos adolescentes do sexo masculino em todas as faixas etárias. A participação dos mais jovens foi sempre maior do que a dos mais velhos, em ambos os sexos.

A Tabela 1 mostra que a maioria da população é composta por indivíduos com cor da pele parda (52,5\%), de escolas públicas $(78,7 \%)$ e estado de peso adequado (72,6\%). A média de idade foi de 14,7 (desvio padrão $=1,6$ ) anos. Menos da metade da amostra $(44,4 \%)$ foi composta por adolescentes ativos ( $\geq 300$ minutos/semana), 29,1\% reportaram nenhuma atividade física no lazer ( 0 minuto/semana), e 26,5\% foram considerados insuficientemente ativos (1-299 minutos/semana). Uma parcela significativa dos jovens não praticava nenhum esporte $(52,4 \%)$. Sobre a educação física na escola, $77,6 \%$ participavam das aulas, sendo que 40,6\% faziam um tempo/semana; 32,6\%, dois tempos/semana; e 4,4\%, três tempos/semana.

Na Tabela 2 pode-se observar a associação dos transtornos mentais comuns com o volume de atividade física no lazer e com a prática esportiva. A frequência de indivíduos com pontuação $\geq 3$ no GHQ-12 foi maior no grupo dos adolescentes que não praticaram esportes $(\mathrm{OR}=1,14)$ e não realizaram nenhuma atividade física no lazer (0 minuto/semana) $(\mathrm{OR}=1,16)$, quando comparados aos jovens ativos.

Atividade física insuficiente (1-299 minutos/semana) não aumentou a razão de chances de transtornos mentais comuns (Tabela 2). Do mesmo modo, não houve aumento significativo no OR após subdivisão dos adolescentes insuficientemente ativos em duas categorias (1-149 minutos/semana e 150-299 minutos/semana). Quando comparados com indivíduos que não praticaram nenhuma atividade física no lazer, os insuficientemente ativos tiveram menores chances de transtornos mentais comuns (OR ajustado = 0,79; IC95\% 0,70-0,89).

A prática de atividade física durante o lazer, independentemente de modalidade, duração ou frequência semanal, reduziu em $26 \%$ as chances de transtornos mentais comuns nessa população (Tabela 2), enquanto a prática em conformidade com a recomendação de pelo menos 300 minutos/semana reduziu em 14\% (ajustado = 0,86; IC95\% 0,79-0,94).

Com relação à prática de educação física na escola (Tabela 3), foi observada relação inversa entre participação nas aulas e os transtornos mentais comuns $(\mathrm{p}<0,01)$. A prevalência de transtornos mentais comuns foi maior entre jovens que não participavam das aulas de educação física escolar (39,5\%), comparados com os que participavam da educação física escolar (29,6\%). 
Tabela 1. Caracterização da amostra de adolescentes brasileiros, segundo variáveis sociodemográficas, maturação sexual e estado de peso. Erica, Brasil, 2013-2014 ( $n$ = 73.399).

\begin{tabular}{|c|c|}
\hline Variáveis & $\%$ \\
\hline \multicolumn{2}{|l|}{ Sexo } \\
\hline Meninos & 44,6 \\
\hline Meninas & 55,4 \\
\hline \multicolumn{2}{|l|}{ Idade (anos) } \\
\hline $12-14$ & 45,9 \\
\hline $15-17$ & 54,1 \\
\hline \multicolumn{2}{|l|}{ Cor da pele } \\
\hline Preta & 7,8 \\
\hline Parda & 52,5 \\
\hline Branca & 36,4 \\
\hline Amarela & 2,6 \\
\hline Indígena & 0,7 \\
\hline \multicolumn{2}{|l|}{ Tipo de escola } \\
\hline Pública & 78,7 \\
\hline Privada & 21,3 \\
\hline \multicolumn{2}{|c|}{ Estágio de maturação sexuala } \\
\hline Pré-púberes & 0,5 \\
\hline Púberes & 62,9 \\
\hline Pós-púberes & 36,6 \\
\hline \multicolumn{2}{|l|}{ Estado de peso ${ }^{b}$} \\
\hline Baixo peso & 2,9 \\
\hline Adequado & 72,6 \\
\hline Sobrepeso & 16,8 \\
\hline Obesidade & 7,7 \\
\hline \multicolumn{2}{|c|}{ Transtornos mentais comuns } \\
\hline Sim & 31,8 \\
\hline Não & 68,2 \\
\hline
\end{tabular}

a Pré-púberes (estágios de Tanner 1 e 2), púberes (estágio de Tanner 3) e pós-púberes (estágios de Tanner 4 e 5).

${ }^{b}$ Classificação do estado nutricional, segundo escore $z$ de índice de massa corporal para a idade ${ }^{33}$.

Tabela 2. Prevalência de adolescentes brasileiros com transtornos mentais comuns/TMC (GHQ $\geq 3$ ) e odds ratios (OR) correspondentes, de acordo com a atividade física no lazer e a prática esportiva. Erica, Brasil, 2013-2014.

\begin{tabular}{|c|c|c|c|c|}
\hline & Total (n) & Com TMC (\%) & OR bruto (IC95\%) & OR ajustado $^{\text {b }}($ IC95\% $\%)$ \\
\hline \multicolumn{5}{|l|}{ Prática de atividade física no lazer ${ }^{c}$} \\
\hline Não & 13.334 & 38,5 & 1,00 (referência) & 1,00 (referência) \\
\hline $\operatorname{Sim}$ & 60.065 & 30,4 & $0,64(0,58-0,70)^{\mathrm{a}}$ & $0,74(0,66-0,82)^{\mathrm{a}}$ \\
\hline \multicolumn{5}{|l|}{ Tempo de atividade física no lazer } \\
\hline Ativos ( $\geq 300$ minutos/semana) & 32.613 & 28,4 & 1,00 (referência) & 1,00 (referência) \\
\hline Insuficientemente ativos (1-299 minutost/semana) & 19,453 & 30,4 & $1,07(0,95-1,19)$ & $0,92(0,82-1,03)$ \\
\hline Inativos (0 minuto/semana) & 21.333 & 38,4 & $1,58(1,46-1,69)^{\mathrm{a}}$ & $1,16(1,06-1,27)^{\mathrm{a}}$ \\
\hline \multicolumn{5}{|l|}{ Prática esportiva ${ }^{d}$} \\
\hline $\operatorname{Sim}$ & 34.932 & 25,9 & 1,00 (referência) & 1,00 (referência) \\
\hline Não & 38.467 & 37,2 & $1,63(1,52-1,74)^{\mathrm{a}}$ & $1,14(1,04-1,25)^{\mathrm{a}}$ \\
\hline
\end{tabular}

IC95\% = intervalo de confiança de 95\%.

a $\mathrm{p}<0,01$.

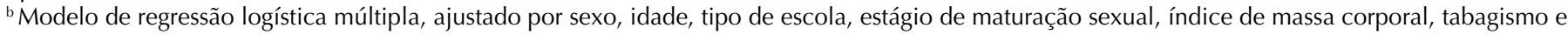
consumo de álcool.

c Prática de alguma atividade física no lazer, independentemente da modalidade, duração ou frequência semanal.

¿ Prática de uma das seguintes modalidades: atletismo, natação, tênis, lutas, ginástica olímpica, futebol/futsal, handebol, basquete e vôlei. 
Tabela 3. Prevalência de adolescentes brasileiros com transtornos mentais comuns/TMC (GHQ $\geq 3$ ) e odds ratios (OR) correspondentes, de acordo com a participação em aulas de educação física na escola. Erica, Brasil, 2013-2014.

\begin{tabular}{|c|c|c|c|c|}
\hline & Total (n) & Com TMC (\%) & OR bruto (IC95\%) & OR ajustado $^{\mathrm{b}}($ IC95\%) \\
\hline \multicolumn{5}{|c|}{ Participação nas aulas de educação física ${ }^{c}$} \\
\hline Sim & 56.920 & 29,6 & 1,00 (referência) & 1,00 (referência) \\
\hline Não & 16.479 & 39,5 & $1,56(1,45-1,68)^{a}$ & $1,25(1,15-1,36)^{a}$ \\
\hline \multicolumn{5}{|c|}{ Frequência da educação física na escola } \\
\hline Não participaram & 16.479 & 39,5 & 1,00 (referência) & 1,00 (referência) \\
\hline 1 tempo por semana & 29.791 & 31,3 & $0,71(0,65-0,77)^{\mathrm{a}}$ & $0,85(0,77-0,93)^{\mathrm{a}}$ \\
\hline 2 tempos por semana & 23.929 & 27,8 & $0,58(0,54-0,63)^{a}$ & $0,74(0,68-0,82)^{a}$ \\
\hline 3 tempos por semana & 3.200 & 27,5 & $0,63(0,53-0,73)^{a}$ & $0,91(0,76-1,09)$ \\
\hline
\end{tabular}

IC95\%= intervalo de confiança de 95\%.

${ }^{a} \mathrm{p}<0,01$

bModelo de regressão logística múltipla, ajustado por sexo, idade, tipo de escola, estágio de maturação sexual, índice de massa corporal, tabagismo e consumo de álcool.

c Participação nas aulas de educação física da escola pelo menos um tempo por semana.

\section{DISCUSSÃO}

Este é o primeiro estudo epidemiológico conduzido no Brasil, com representatividade nacional, que avaliou a relação entre transtornos mentais comuns e níveis de atividade física durante a adolescência. A pesquisa produziu três resultados para discussão. Primeiro, a frequência de transtornos mentais comuns foi maior entre adolescentes que não praticavam esportes, não participavam das aulas de educação física na escola e eram inativos (0 minuto/semana) no lazer. Segundo resultado, atividade física insuficiente (1-299 minutos/semana) não aumentou a razão de chances de transtornos mentais comuns nessa população. Terceiro, foi observado que a prática de atividade física em conformidade com a recomendação de pelo menos 300 minutos/semana reduziu a razão de chances para os transtornos mentais comuns, contudo não ofereceu benefício adicional para a saúde mental dos jovens. Redução significante na chance de transtornos mentais comuns foi observada com atividade física no lazer independentemente de modalidade, duração e frequência semanal.

As poucas evidências atualmente disponíveis sobre o assunto têm mostrado o impacto negativo de atividade física insuficiente na saúde mental da população jovem ${ }^{19,20}$. Estudo realizado na Noruega, com adolescentes de 12 a 15 anos, mostrou relação transversal e longitudinal entre baixos níveis de atividade vigorosa e sintomas depressivos (usando o Mood and Feelings Questionnaire) ${ }^{19}$. Coorte prospectiva (2004-2010), conduzida na Dinamarca, apresentou resultados semelhantes, mas somente para o sexo feminino. Meninas com baixo nível de atividade física no lazer aos 14/15 anos tiveram risco aumentado em $60 \%$ para problemas de saúde mental aos 20/21 anos, comparadas com as com alto nível (> 4 horas/semana ${ }^{20}$.

Os resultados da presente pesquisa condizem com a revisão sistemática de estudos prospectivos envolvendo crianças, adolescentes e adultos (1988-2012), que sugeriram qualquer nível de atividade física, como caminhar menos de 150 minutos/semana, para prevenir depressão (definida por pontos de corte em escalas de autorrelato ou diagnóstico médico $)^{21}$. Pesquisa recente também forneceu apoio encorajador para a atividade física enquanto intervenção benéfica no humor. Trinta minutos de corrida em intensidade moderada, durante a manhã, por três semanas consecutivas, impactaram positivamente na concentração durante o dia e no funcionamento psicológico de adolescentes, em comparação com indivíduos controle ${ }^{22}$.

Efeito benéfico similar foi observado em estudo com adolescentes australianos, que evidenciou um decréscimo de $9 \%$ na probabilidade de sintomas depressivos entre os meninos que praticavam atividades moderadas a vigorosas, diariamente, por mais de uma hora ${ }^{6}$. Alghadir e colaboradores ${ }^{23}$, estudando indivíduos de 7 a 18 anos, concluíram que a prática de atividade física é importante para a melhora dos sintomas depressivos (escala Children's Depression Inventory), além de aumentar os níveis de serotonina e reduzir o cortisol sérico. 
Esse estudo apresentou associação positiva entre atividade física no lazer e saúde mental, independentemente de modalidade, duração ou frequência da prática. Assim, as políticas públicas poderiam considerar a inclusão da dimensão saúde mental dos jovens totalmente inativos (0 minuto/semana),já que parece não existir benefício adicional para a saúde mental associado ao cumprimento dos níveis de atividade recomendados pela OMS. Cabe ressaltar que há evidências científicas de que a saúde física dos adolescentes é melhorada por atividade física frequente ${ }^{24}$.

Os achados do presente estudo evidenciaram maior ocorrência de transtornos mentais comuns na parcela da população que não praticou esportes. Corroborando nosso estudo, Sabiston e colaboradore ${ }^{25}$ constataram que a participação em esportes de equipe durante o ensino médio pode proteger contra os sintomas depressivos no início da vida adulta, provavelmente pelo fato de o esporte em equipe proporcionar trabalho em grupo, oportunidade de interação com pessoas e melhor aceitação social percebida. Atividade física no lazer e participação em esportes também contribuíram para uma maior sensação de bem-estar e menores níveis de ansiedade e depressão entre adolescentes europeus ${ }^{26}$.

Com relação a atividade física na escola, foi observado que a participação em aulas de educação física foi negativamente associada a transtornos mentais comuns na amostra do Erica. Estudo transversal com adolescentes australianos (10-16 anos) detectou, do mesmo modo, que ser mais ativo na escola, participando da educação física (OR = 0,77; IC95\% $0,69-0,86)$ e das equipes de esportes $(\mathrm{OR}=0,77$; IC95\% 0,67-0,88), pode diminuir a razão de chances para os sintomas depressivos ${ }^{27}$. Nessa perspectiva, a escola é um espaço privilegiado para adolescentes realizarem atividades físicas, considerando que é um local seguro, com equipamentos disponíveis e orientação profissional apropriada ${ }^{9}$. Contudo, é importante destacar a ausência de uma relação dose-resposta clara entre o nível de atividade física e a presença de transtornos mentais comuns.

A relação entre bem-estar mental e atividade física tem atraído atenção de pesquisadores, entretanto alguns estudos não apresentaram associações consistentes. De acordo com Rothon e colaboradore ${ }^{28}$, cada hora adicional de atividade física empreendida na semana diminui em cerca de $8 \%$ a probabilidade de sintomas depressivos na adolescência. Todavia, a relação entre essas variáveis não foi significante em uma análise longitudinal, após dois anos de acompanhamento. Estudo de coorte no Reino Unido também não sustentou a hipótese de que atividade física, objetivamente mensurada por frequência cardíaca e detecção de movimento, evita o desenvolvimento de sintomas depressivos (Mood and Feelings Questionnaire) durante a adolescência ${ }^{29}$.

Várias hipóteses têm sido propostas para explicar o efeito protetor da atividade física. Mecanismos biológicos, como o aumento dos níveis de monoaminas na circulação (norepinefrina, dopamina e serotonina) e menor secreção de cortisol, podem explicar a ação antidepressiva da atividade física ${ }^{23}$. Além disso, o cumprimento de metas e desafios da atividade motora provoca o sentimento de autoeficácia, melhora a autoestima e gera distração dos estressores diários ${ }^{19}$. A rede social construída na atividade esportiva também pode ser importante, assim como o comportamento sedentário pode reduzir as interações sociais entre os indivíduos, gerando sentimentos de solidão ${ }^{30}$.

De outro modo, sintomas depressivos, mesmo abaixo do limiar de diagnóstico clínico, apatia e sentimentos de desesperança podem iniciar um padrão negativo em que é difícil reunir energia e motivação para iniciar a prática de atividade física ${ }^{19}$. O efeito protetor da atividade física e o efeito inibitório do humor deprimido também podem coexistir. Dados de um estudo longitudinal com meninas de 11 a 15 anos, avaliadas anualmente por um período de seis anos, sinalizaram uma relação bidirecional entre atividade física e depressão. A prática de atividade física reduziu significantemente tanto o risco de transtorno depressivo maior como o risco de sintomas depressivos futuros, e vice-versa ${ }^{31}$.

Uma das limitações deste estudo foi o uso de um questionário para determinar o nível de atividade física dos adolescentes. Apesar desse instrumento ter sido validado ${ }^{15}$, nem sempre 
produz estimativas precisas, e sua concordância com medidas diretas é parcial ${ }^{32}$. Contudo, a utilização de métodos de mensuração direta, como acelerometria, seria inviável em um estudo como o Erica, devido ao alto custo e à complexidade da logística envolvida. A variedade de instrumentos e pontos de corte (para mensurar os transtornos mentais comuns e os níveis de atividade física) na literatura científica também limitou o presente estudo, dificultando a comparação com nossos resultados. O GHQ-12 é uma ferramenta de triagem, e, por isso, não foi possível estabelecer diagnóstico formal de psicopatologias.

Outra limitação está relacionada à natureza transversal (causalidade reversa). Vale ressaltar, ainda, que o uso do OR pode superestimar a força da associação em estudos de delineamento transversal, quando o desfecho possui elevada prevalência na população, o que possivelmente ocorreu no presente estudo. Além disso, outra questão que coloca em discussão a plausibilidade da associação possuir relação causal é a relativamente pequena magnitude da associação. O poder da amostra poderia identificar magnitudes inclusive menores que as encontradas no presente estudo, o que faz refletir sobre a relevância/propriedade de se fazer recomendações mais incisivas. Por outro lado, os pontos fortes deste trabalho incluem a amostra grande e representativa para os adolescentes brasileiros, a utilização de questionários validados, a alta taxa de participação e as análises ajustadas por diversos fatores de confusão (sexo, idade, tipo de escola, estágio de maturação sexual, índice de massa corporal, tabagismo e uso de álcool).

Em conclusão, transtornos mentais comuns estão associados a inatividade física no lazer (0 minuto/semana) e na escola durante a adolescência. Atividade física no tempo de lazer, mesmo sem atingir a recomendação atual para a saúde ( $\geq 300$ minutos/semana), pode reduzir a razão de chances de transtornos mentais comuns, de acordo com os dados do presente estudo. Prática esportiva e educação física escolar também podem ter efeitos benéficos na saúde mental dos jovens. Os resultados do Erica reforçam a necessidade de realizar a transição da inatividade total para uma prática regular de atividade física, independentemente da frequência semanal e do volume de atividade física inicialmente praticada.

\section{REFERÊNCIAS}

1. Belfer ML. Child and adolescent mental disorders: the magnitude of the problem across the globe. J Child Psychol Psychiatry. 2008;49(3):226-36. https://doi.org/10.1111/j.1469-7610.2007.01855.x.

2. Gore FM, Bloem PJ, Patton GC, Ferguson J, Joseph V, Coffey C et al. Global burden of disease in young people aged 10-24 years: a systematic analysis. Lancet. 2011;377(9783):2093-102. https://doi.org/10.1016/S0140-6736(11)60512-6.

3. Lopes CS, Abreu GA, Santos DF, Menezes PR, Carvalho KMB, Cunha CF et al. Erica: prevalence of common mental disorders in Brazilian adolescents. Rev Saude Publica. 2016;50(1):14s. http://dx.doi.org/10.1590/s01518-8787.2016050006690.

4. Goldberg DP, Williams P. A user's guide to the General Health Questionnaire - GHQ. Windsor: Nfer-Nelson; 1988.

5. Goldberg DP, Gater R, Sartorius N, Ustun TB, Piccinelli M, Gureje O et al. The validity of two versions of the GHQ in the WHO study of mental illness in general health care. Psychol Med. 1997;27(1):191-7.

6. Hayward J, Jacka FN, Skouteris H, Millar L, Strugnell C, Swinburn BA et al. Lifestyle factors and adolescent depressive symptomatology: Associations and effect sizes of diet, physical activity and sedentary behavior. Aust N Z J Psychiatry. 2016;50(11):1064-73. http://dx.doi.org/10.1177/0004867416671596.

7. Langguth N, Schmid J, Gawrilow C, Stadler G. Within-person link between depressed affect and moderate-to-vigorous physical activity in adolescence: an intensive longitudinal approach. Appl Psychol Health Well Being. 2016;8(1):44-63. http://dx.doi.org/10.1111/aphw.12061.

8. Bélanger M, Sabiston CM, Barnett TA, O'Loughlin E, Ward S, Contreras G et al. Number of years of participation in some, but not all, types of physical activity during adolescence predicts level of physical activity in adulthood: results from a 13-year study. Int J Behav Nutr Phys Act. 2015;12:76. http://dx.doi.org/10.1186/s12966-015-0237-x. 
9. Cureau FV, Silva TLN, Bloch KV, Fujimori E, Belfort DR, Carvalho KMB et al. Erica: leisure-time physical inactivity in Brazilian adolescents. Rev Saude Publica. 2016;50(1):4s. http://dx.doi.org/10.1590/s01518-8787.2016050006683.

10. WHO. Global health risks: mortality and burden of disease attributable to selected major risks. Genebra: World Health Organization; 2009.

11. Gobbi E, Greguol M, Carraro A. Brief report: exploring the benefits of a peer-tutored physical education programme among high school students with intellectual disability. J Appl Res Intellect Disabil. 2018;31(5):937-41. http://dx.doi.org/10.1111/jar.12437.

12. Bloch KV, Szklo M, Kuschnir MCC, Abreu GA, Barufaldi LA, Klein CH et al. The study of cardiovascular risk in adolescents - Erica: rationale, design and sample characteristics of a national survey examining cardiovascular risk factor profile in Brazilian adolescents. BMC Public Health. 2015;15:94. http://dx.doi.org/10.1186/s12889-015-1442-x.

13. Tanner JM. Growth at adolescence. $2^{\text {nd }}$ ed. Oxford: Blackwell Scientific; 1962.

14. Sallis JF, Strikmiller PK, Harsha DW, Feldman HA, Ehlinger S, Stone EJ et al. Validation of interviewer- and self-administered physical activity checklists for fifth grade students. Med Sci Sports Exerc. 1996;28(7):840-51.

15. Farias Júnior JC, Lopes AS, Mota J, Santos MP, Ribeiro JC, Hallal PC. Validity and reproducibility of a physical activity questionnaire for adolescents: adapting the Self-Administered Physical Activity Checklist. Rev Bras Epidemiol. 2012;15(1):198-210.

16. WHO. Global recommendations on physical activity for health. Genebra: World Health Organization; 2010.

17. Currie $C$ et al. Inequalities in young people's health: HBSC international report from the 2005/2006 survey. Copenhagen: World Health Organization; 2008. Health policy for children and adolescents, n. 5 .

18. Mari JJ, Williams P. A comparison of the validity of two psychiatric screening questionnaires (GHQ-12 and SRQ-20) in Brazil, using relative operating characteristic (ROC) analysis. Psychol Med. 1985;15(3):651-9.

19. Sund AM, Larsson B, Wichstrom L. Role of physical and sedentary activities in the development of depressive symptoms in early adolescence. Soc Psychiatry Psychiatr Epidemiol. 2011;46(5):431-41. http://dx.doi.org/10.1007/s00127-010-0208-0.

20. Poulsen $\mathrm{PH}$, Biering $\mathrm{K}$, Andersen $\mathrm{JH}$. The association between leisure time physical activity in adolescence and poor mental health in early adulthood: a prospective cohort study. BMC Public Health. 2016;16(3):1-11. http://dx.doi.org/10.1186/s12889-015-2658-5.

21. Mammen G, Faulkner G. Physical activity and the prevention of depression: a systematic review of prospective studies. Am J Prev Med. 2013;45(5):649-57. http://dx.doi.org/10.1016/j.amepre.2013.08.001.

22. Kalak N, Gerber M, Kirov R, Mikoteit T, Yordanova J, Pühse $U$ et al. Daily morning running for 3 weeks improved sleep and psychological functioning in healthy adolescents compared with controls. J Adolesc Health. 2012;51(6):615-22. http://dx.doi.org/10.1016/j.jadohealth.2012.02.020.

23. Alghadir AH, Gabr SA, Al-Eisa E. Effects of physical activity on trace elements and depression related biomarkers in children and adolescents. Biol Trace Elem Res. 2016;172(2):299-306. http://dx.doi.org/10.1007/s12011-015-0601-3.

24. Hallal PC, Victora CG, Azevedo MR, Wells JC. Adolescent physical activity and health: a systematic review. Sports Med. 2006;36(12):1019-30. http://dx.doi.org/10.2165/00007256-200636120-00003.

25. Sabiston CM, Jewett R, Ashdown-Franks G, Belanger M, Brunet J, O'Loughlin E et al. Number of years of team and individual sport participation during adolescence and depressive symptoms in early adulthood. J Sport Exerc Psychol. 2016;38(1):105-10. http://dx.doi.org/10.1123/jsep.2015-0175.

26. McMahon EM, Corcoran P, O'Regan G, Keeley H, Cannon M, Carli V et al. Physical activity in European adolescents and associations with anxiety, depression and well-being. Eur Child Adolesc Psychiatry. 2017;26(1):111-22. http://dx.doi.org/10.1007/s00787-016-0875-9.

27. Kremer P, Elshaug C, Leslie E, Toumbourou JW, Patton GC, Williams J. Physical activity, leisure-time screen use and depression among children and young adolescents. J Sci Med Sport. 2014;17(2):183-7. http://dx.doi.org/10.1016/j.jsams.2013.03.012. 
28. Rothon C, Edwards P, Bhui K, Viner RM, Taylor S, Stansfeld SA. Physical activity and depressive symptoms in adolescents: a prospective study. BMC Med. 2010;8:32. http://dx.doi.org/10.1186/1741-7015-8-32.

29. Toseeb U, Brage S, Corder K, Dunn VJ, Jones PB, Owens M et al. Exercise and depressive symptoms in adolescents: a longitudinal cohort study. JAMA Pediatr. 2014;168(12):1093-100. http://dx.doi.org/10.1001/jamapediatrics.2014.1794.

30. Kraut R, Patterson M, Lundmark V, Kiesler S, Mukopadhyay T, Scherlis W. Internet paradox: a social technology that reduces social involvement and psychological well-being?. Am Psychol. 1998;53(9):1017-31.

31. Jerstad SJ, Boutelle KN, Ness KK, Stice E. Prospective reciprocal relations between physical activity and depression in female adolescents. J Consult Clin Psychol. 2010;78(2):268-72. http://dx.doi.org/10.1037/a0018793.

32. Steene-Johannessen J, Anderssen SA, Ploeg HP, Hendriksen IJ, Donnelly AE, Brage S et al. Are self-report measures able to define individuals as physically active or inactive?. Med Sci Sports Exerc. 2016;48(2):235-44. http://dx.doi.org/10.1249/MSS.0000000000000760.

33. Organização Mundial de Saúde. Growth reference data for 5-19 years. 2007. [citado 23 out 2020]. Disponível em: https://www.who.int/growthref/en/.

Financiamento: Conselho Nacional de Desenvolvimento Científico e Tecnológico (CNPq - Processos 565037/2010-2 e 405009/2012-7.

Contribuição dos Autores: Concepção do estudo, análise dos dados, redação do artigo: VRF. TVJ Revisão do artigo quanto ao conteúdo intelectual: TVJ, TIRP, RBV, ALLS, PCVJ. Aquisição de financiamento: PCVJ. Todos os autores leram e aprovaram a versão final do manuscrito.

Conflito de Interesses: Os autores declaram não haver conflito de interesses. 